\title{
Effects of motor preparation and spatial attention on corticospinal excitability in a delayed-response paradigm
}

\author{
Rogier B. Mars · Sven Bestmann · John C. Rothwell • \\ Patrick Haggard
}

Received: 5 April 2007 / Accepted: 27 June 2007 / Published online: 19 July 2007

(C) Springer-Verlag 2007

\begin{abstract}
The preparation of motor responses during the delay period of an instructed delay task is associated with sustained neural firing in the primate premotor cortex. It remains unclear how and when such preparation-related premotor activity influences the motor output system. In this study, we tested modulation of corticospinal excitability using single-pulse transcranial magnetic stimulation (TMS) during a delayed-response task. At the beginning of the delay interval participants were either provided with no information, spatial attentional information concerning location but not identity of an upcoming imperative stimulus, or information regarding the upcoming response. Behavioral data indicate that participants used all information available to them. Only when information concerning the upcoming response was provided did corticospinal excitability show differential modulation for the effector muscle compared to other task-unrelated muscles. We conclude that modulation of corticospinal excitability reflects specific response preparation, rather than non-specific event preparation.
\end{abstract}

R. B. Mars $\cdot$ S. Bestmann $\cdot$ J. C. Rothwell

Sobell Department of Motor Neuroscience

and Movement Disorders, Institute of Neurology,

University College London, London, WC1N 3BG, UK

R. B. Mars $(\bowtie) \cdot$ P. Haggard

Institute of Cognitive Neuroscience, University College London,

17 Queen Square, London, WC1N 3AR, UK

e-mail: r.mars@ucl.ac.uk

S. Bestmann

Wellcome Trust Centre for Neuroimaging, Institute of Neurology,

University College London, London, WC1N 3BG, UK

P. Haggard

Department of Psychology, University College London,

London, WC1E 6BT, UK
Keywords Movement preparation - Spatial attention . Delayed-response task · Corticospinal excitability · TMS . Motor preparation $\cdot$ Response preparation

\section{Introduction}

Humans and other primates are able to use prior information to prepare their motor system for a later response (Rosenbaum 1980). This motor preparation is evident in a shorter reaction time and is reflected in preparatory neural activity. An important question to ask when trying to understand the nature of preparatory neural processes is with what type of information a certain neural structure is dealing. For instance, in the domain of arbitrary or symbolic visuomotor associations (Wise and Murray 2000), the dorsal premotor cortex (PMd) has consistently been shown to exhibit strong preparatory activity (e.g., Kurata and Wise 1988; Toni et al. 1999). However, the type and locus of activity within PMd differs depending on the amount of information concerning the upcoming movement that is available to the participant (Hoshi and Tanji 2000; Mars et al. 2005). When preparatory information is given about only some attributes of the imperative stimulus, activity can be found in the rostral part of PMd. Conversely, when the information about the imperative stimulus is sufficient to specify the response in advance, preparatory activity is present in the most caudal parts of PMd, which have access to the primary motor cortex and the spinal cord (Mars et al. 2005). Consistent with this finding, modulation of activity of spinal neurons during movement preparation has been observed in monkeys (Prut and Fetz 1999).

Studies probing human corticospinal excitability in delayed-response paradigms using transcranial magnetic stimulation (TMS), however, have yielded inconsistent 
results. A number of studies have reported a decrease in corticospinal excitability during instructed delay periods, but no response-specific change in corticospinal excitability (e.g., Touge et al. 1998; Hasbroucq et al. 1999). Conversely, some recent studies reported increases in corticospinal excitability, specific to the prepared response (Van den Hurk et al. 2007; Van Elswijk et al. 2007). These conflicting results can be partly explained by differences across studies in the timing of the response, and therefore in the degree of preparation, or in the predictability of the TMS pulse. Both of these factors have been shown to influence corticospinal excitability (Van Elswijk et al. 2007; Takei et al. 2005). Interestingly, Van den Hurk et al. 2007 employed a range of delay periods (varying between 1 and $9 \mathrm{~s}$ ) resulting in a low predictability of both response time and timing of the TMS pulse. However, these types of delays are quite unusual in preparation studies using TMS and may result in fluctuations in the level of motor preparation during the delay period.

In the present study, we aim to investigate whether a specific increase in corticospinal excitability with selective response preparation can be found in a delayed response paradigm with delays in the range normally studied using TMS. Furthermore, we employ spatially compatible cues to instruct movement preparation. Since previous studies (e.g., Van den Hurk et al. 2007) cued movement preparation using arbitrary cues (cf. Wise and Murray 2000), it remains to be seen whether these results generalize to other types of movement cueing.

Participants were required to respond by pressing a button with either the left or right hand in response to a trigger cue. The trigger cue was presented either to the left of the right side of fixation and consisted of a symbolic cue instructing one of the two possible responses. At the beginning of each trial, an instruction cue could give participants prior information on the location of the upcoming trigger cue or the movement it would instruct. We probed corticospinal excitability during the delay period between the instruction and the trigger cues using single-pulse transcranial magnetic stimulation. This allowed us to investigate whether the processing of advance motor information has distinct effects on corticospinal excitability over and above effects of prior information and general preparation. Critically, the timing of both response time and TMS pulse varied and was equally (un)predictable between conditions.

\section{Materials and methods}

\section{Participants}

Eleven healthy right-handed volunteers (4 women; age range $18-31$ years) participated in the experiment. All participants gave their informed consent prior to participation. Experimental procedures were approved by the local ethics committee and performed according to the ethical standards laid down in the Declaration of Helsinki. No participants reported any adverse effects of TMS. The data of the last two (out of four) experimental blocks of one participant were discarded due to excessive head movements.

\section{Electromyographic recording}

Surface electromygraphic (EMG) was recorded from the right first dorsal interosseous (FDI) muscle with a bellytendon montage using $\mathrm{Ag} / \mathrm{AgCl}$ surface electrodes. Raw signals were amplified using Cambridge Electronic Design 1902 amplifiers with a band-pass filter of 1-2,000 Hz. Signals were stored on a personal computer for later analysis at a sampling rate of $5 \mathrm{kHz}$. Participants were asked to maintain relaxation of the target muscle throughout the experiment.

\section{Stimulation procedure}

Transcranial magnetic stimulation was applied over the hand area of the left motor cortex with a figure-of-eight coil (70 mm outer wing diameter) using a Magstim 200 stimulator (Magstim Co. Whitland, UK). The coil was held tangentially over the left side of the scalp with its handle pointing backwards at an angle of approximately $45^{\circ}$ from the midsagittal axis. The site of stimulation was optimal to elicit motor-evoked potentials (MEPs) in the right FDI muscle. The coil position was marked on the participants head so that it could be maintained at that location during the experiment. Stimulation intensity for the main experiment was set to evoke an MEP of $1 \mathrm{mV}$ peak-to-peak amplitude, corresponding to a stimulator output of 30-60\% for all participants.

\section{Experimental procedure}

The experiment consisted of a training and an experimental part. During the first training session (30 trials), participants were trained on the associations between two visual stimuli and two motor responses (Fig. 1a). Motor responses consisted of button presses with the index finger of the left and right hands, respectively. On each trial, participants were presented with one of two visual shapes (300 ms, centrally presented). Following a variable delay $(1,500-2,000 \mathrm{~ms})$ participants heard an auditory beep $(1,000 \mathrm{~Hz}, 300 \mathrm{~ms})$, after which they responded as fast as possible to the movement instructed by the visual shape. Visual feedback was presented centrally $200 \mathrm{~ms}$ later to indicate correct ("V"), incorrect ("X"), or too late ("!") responses. 
Fig. 1 Experimental protocol. a Stimulus-response associations. b Trial structure. At the beginning of each trial participants received a neutral, spatial attentional, or motor instructional cue. On half the trials, a single TMS pulse was delivered $200 \mathrm{~ms}$ before the onset of the trigger cue. The trigger stimulus was presented 1,500-2,000 ms following instruction cue onset to the left or the right of fixation
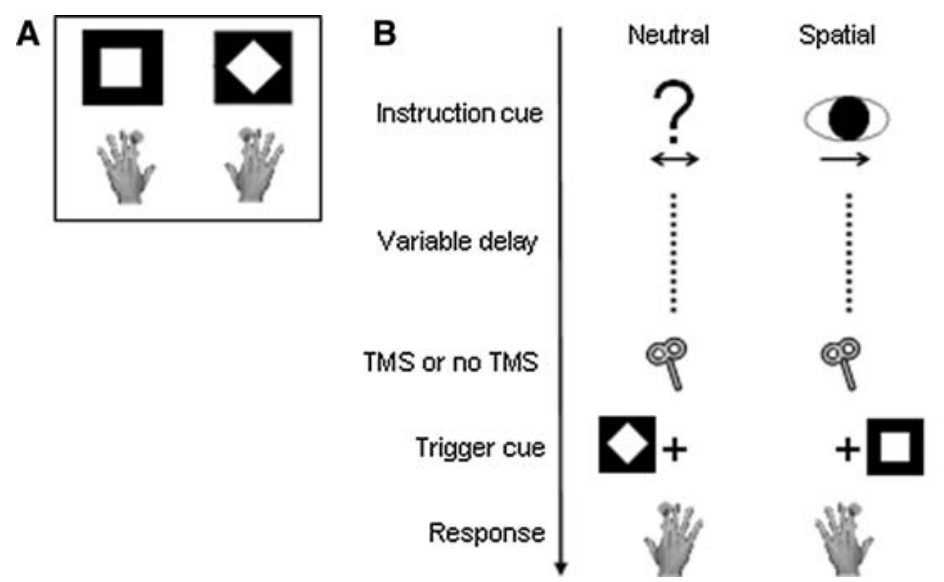

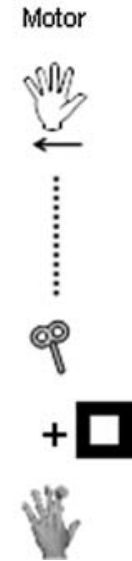

In a second training session, participants were familiarized with the main experimental task (Fig. 1b). Participants fixated on a cross at the centre of the screen throughout the task. At trial onset, a central instruction cue was presented for $300 \mathrm{~ms}$, followed after a delay (varying between 1,500 and $2,000 \mathrm{~ms}$, uniform distribution) by a target stimulus ("trigger cue", $200 \mathrm{~ms}$ ). The trigger cue was one of the previously trained visual shapes and was presented to the left or right of the fixation cross at approximately $14.5^{\circ}$ visual angle. Participants were instructed to press the button associated with the visual shape as quickly as possible.

The instruction cue distinguished between the three experimental conditions: (1) Neutral: a question mark with an arrow pointing both left and right, providing no information regarding the forthcoming trigger cue; (2) Spatial: a picture of an eye with an arrow pointing to the left or the right indicating with $100 \%$ validity where the subsequent trigger cue would appear; or (3) Motor: a hand with an arrow pointing to the left or the right indicating with $100 \%$ validity, which response (left or right press) the subsequent trigger cue would require.

Consequently, the three different conditions provided different degrees of certainty regarding the required motor response. Participants had either no advance information (NEUTRAL), information regarding the spatial location of the imperative stimulus (SPATIAL), or full information regarding the required motor response irrespective of the spatial location of the trigger stimulus (мОтоR). Participants trained each of the different conditions separately for 16 trials each. They then performed one block of 50 trials with all three conditions intermixed. Performance feedback was provided as in the first training session.

An additional training block of 50 trials was performed without performance feedback, and with TMS applied unpredictably on $50 \%$ of trials. The TMS pulse was applied $200 \mathrm{~ms}$ prior to the presentation of the trigger cue, to ensure that any movement preparation was maximal at the moment of stimulation. Following the training block, reaction times were inspected to ensure similar performance with and without TMS being present. If necessary, this procedure was repeated until participants were able to perform the task equally well independent of the presence of TMS.

Four experimental blocks of 50 trials each were then run. Each block contained 10 NEUTRAL trials, 20 SPATIAL trials (10 cueing the left side, 10 cueing the right side), and 20 MOTOR trials (10 cueing left hand movement, 10 cueing right hand movement). On half of the trials, participants received a single TMS pulse $200 \mathrm{~ms}$ prior to presentation of the trigger cue. Both trial type and application of TMS were randomized.

Data analysis

Trials with incorrect, multiple, or premature $(\mathrm{RT}<80 \mathrm{~ms})$ responses were discarded from the analysis. Reaction times for correct trials were analyzed in a two-way repeated measures ANOVA with factors TMS (two levels: TMS and no TMS) and CONDITION (three levels: NEUTRAL, SPATIAL, and MOTOR cues).

Peak-to-peak amplitude of each MEP was quantified using custom software in Matlab (The Mathworks, Natick, MA). Trials, in which participants failed to relax the target muscle fully prior to the TMS pulse (maximum EMG amplitude $>75 \mu \mathrm{V}$ in the $200 \mathrm{~ms}$ prior to the TMS pulse) were discarded from the analysis. The size of MEPs was expressed as the percentage of the mean MEP amplitude in the NEUTRAL condition in each block in order to account for between-block differences. MEPs were analyzed in a twoway repeated measures ANOVA with factors CONDITION (2 levels: SPATIAL and MOTOR cues) and SIDE (2 levels: left and right). For the RT and MEPs analyses, significant effects in the ANOVAs were taken as justification for posthoc paired-samples two-tailed $t$-tests.

Although we discarded trials in which there was precontraction of the muscle (see above) we further assessed whether participants were able to relax the target muscle during the delay interval by calculating the root mean square EMG signal recorded in the interval $200-50 \mathrm{~ms}$ 
prior to the TMS pulse. As with the MEP amplitude, this value was normalized to the mean root mean square value of the NEUTRAL condition in each block.

\section{Results}

Behavioral results

Participants made few errors during the experiment, averaging $97.5 \%$ correct responses. Reaction times on correct trials (Fig. 2a) decreased with increasing information regarding the required motor response (main effect of CONDITION: $\left.F_{(2,20)}=61.72, P<0.001\right)$. Post-hoc pairedsamples two-tailed $t$-tests revealed that participants responded faster in the SPATIAL as compared the NEUTRAL condition $\left(t_{(10)}=7.128, P<0.001\right)$ and quicker in the MOTOR as compared to the SPATIAL condition $\left(t_{(10)}=4.781\right.$, $P<0.001)$.

Participants responded quicker on trials with TMS than trials without TMS (main effect of TMS: $F_{(1,10)}=8.87$, $P<0.014)$. However, this speeding did not differ between conditions (CONDITION $\times$ TMS interaction: $F<1$, $P=0.40$ ), indicating that the TMS pulse did not differentially influence the behavioral processes under study.

\section{Motor-evoked potentials}

The main finding of this study was that MEP amplitude in the right FDI (Fig. 2b) was modulated as a function of whether participants were given prior information regarding the upcoming motor response, but not when participants were merely cued to allocate spatial attention. Specifically, the results showed an effect of $\operatorname{SIDE}\left(F_{(1,10)}=17.54, P=0.002\right)$, but only following MOTOR cues (CONDITION $\times$ SIDE interaction: $\left.F_{(1,10)}=21.23, P=0.001\right)$. Post-hoc paired-samples two-tailed $t$-test revealed that MEPs differed between left and right cues in the MOTOR $\left(t_{(10)}=4.77, P=0.001\right)$, but not in the SPATIAL condition $\left(t_{(10)}=1.00, P=0.34\right)$. Following right hand MOTоR cues, MEPs were significantly greater than following NEUTRAL (baseline) cues $\left(t_{(10)}=4.55, P=0.001\right)$. Conversely, MEPs were smaller following left hand мотоR cues than following NeUTRAL cues $\left(t_{(10)}=2.67, P=0.023\right)$. This difference in MEPs in the мотоR cueing condition was not due to a difference in delay period EMG, since this did not differ between left and right cued trials $\left(t_{(10)}=1.17\right.$, $P=0.27$ ).

To ensure that our effects are not due to differential eye movements between conditions, we recorded EOGs in two additional participants performing the same task as during the experimental session in an attempt to find systematic differences in eye movements between conditions. No such differences were found, indicating that our results cannot be explained by any eye movement effects.

\section{Discussion}

In this study we have examined the modulation of corticospinal excitability during a delayed-response task while participants were given different types of prior information. Reaction times indicated that participants used the information presented to them at the beginning of each trial. Compared to the neutral condition, faster responses were given when prior information concerning the stimulus location was given. When information on the response to be executed was given, participants were even faster. Corticospinal excitability was modulated when participants were able to fully prepare their response. Excitability of the muscles used for executing the response (prime mover) was increased while that of the muscle not used in that particular trial was decreased compared to baseline. This pattern of modulation was not found when participants were given only spatial attentional information.

Although some studies have shown an increase in corticospinal excitability during movement preparation (e.g., Van den Hurk et al. 2007; Van Elswijk et al. 2007), the results have been mixed with some studies finding no effect
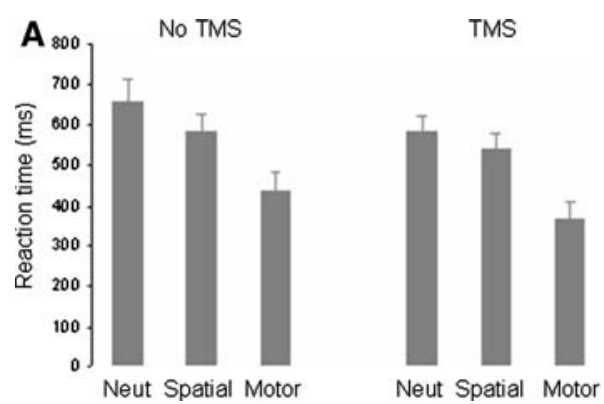

Fig. 2 Experimental results. a Reaction times in NEUTRAL, SPATIAL, and MOTOR conditions separated for trials without (left) and with (right) TMS. b MEP amplitudes in the right FDI muscle on trials

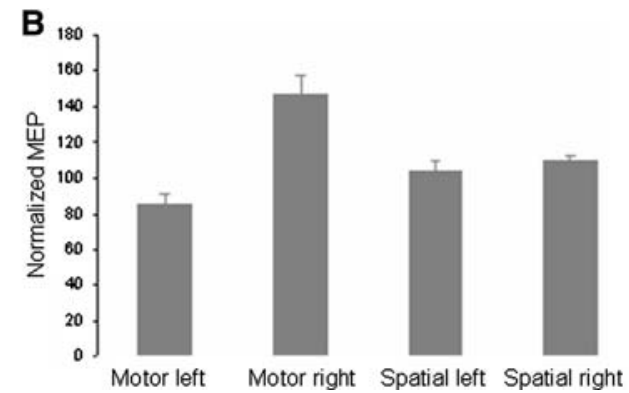

involving left and right cues in the MOTOR and SPATIAL conditions, normalized to the mean of the MEP in the NEUTRAL condition. Error bars indicate SEM 
(Young et al. 2000) or even a decrease in corticospinal excitability (Touge et al. 1998; Hasbroucq et al. 1999) during instructed delay periods. As discussed in the" Introduction", these conflicting results might be partly explained by differences across studies in the timing of the response, and therefore in the degree of preparation (Van Elswijk et al. 2007), or in the timing of the TMS pulse (Takei et al. 2005). In this study, the timing of the TMS pulses was not predictable, since pulses were given randomly on only half the trials and the length of the delay period was variable. Furthermore, in contrast to some previous studies, our design allowed us to distinguish three distinct aspects of preparation. These are the non-specific, general arousal associated with event-preparation, spatial attention to the trigger stimulus, and specific preparation for the motor response. Because these different levels of preparation were defined by differences between conditions, rather than by different times in the time-course of the trial, our results were not confounded by the anticipation of the TMS pulse or differences in timing predictability between conditions.

Another important difference between this study and previous studies is in the type of movement cueing. Previous studies showing response-specific modulation of corticospinal excitability used arbitrary cues to instruct movement preparation (Van den Hurk et al. 2007). However, movement information can reach the movement system via various neural pathways, depending on whether the instruction is provided using arbitrary or spatial cues (e.g., Toni et al. 2001). Here, we show that corticospinal excitability is modulated in a similar manner, independent of the type of cues used.

We conclude that corticospinal excitability of the prime mover is specifically modulated by the preparation of a motor response. These effects are consistent with reports of different loci in premotor cortex associated with processing stimulus and motor information (Boussaoud 2001; Mars et al. 2005). Moreover, these effects generalize across different types of movement instruction, whether the movements are instructed spatially as in this study or using arbitrary visuomotor associations as in previous work (Van den Hurk et al. 2007).
Acknowledgments R.B.M. and S.B. are supported by the Wellcome Trust (Grant number 076358/Z/05/Z).

\section{References}

Boussaoud D (2001) Attention versus intention in the primate premotor cortex. NeuroImage 14:S40-S45

Hasbroucq T, Kaneko H, Akamatsu M, Possamai CA (1999) The timecourse of preparatory spinal and cortico-spinal inhibition: an H-reflex and transcranial magnetic stimulation study in man. Exp Brain Res 124:33-41

Hoshi E, Tanji J (2000) Integration of target and body-part information in the premotor cortex when planning action. Nature 408:466470

Kurata K, Wise SP (1988) Premotor cortex of rhesus monkeys: set-related activity during two conditional motor tasks. Exp Brain Res 69:327-343

Mars RB, Coles MGH, Hulstijn W, Toni I (2005) Sensory and motor delay-related responses in the human brain. Program No. 537.26. 2005 Abstract viewer and itinerary planner. Society for Neuroscience, Washington DC. Online

Prut Y, Fetz EE (1999) Primate spinal interneurons show pre-movement instructed delay activity. Nature 401:590-594

Rosenbaum DA (1980) Human movement intention: specification of arm, direction, and extent. J Exp Psychol Gen 109:444-474

Takei T, Hashimoto T, Hagura N, Matsumura M, Naito E (2005) Reduction of cortico-spinal excitability by transcranial magnetic stimulation at predictable timing. Jpn J Physiol 66:93-99

Toni I, Schluter ND, Josephs O, Friston K, Passingham RE (1999) Signal-, set-, and movement-related activity in the human brain: an event-related fMRI study. Cereb Cortex 9:35-49

Toni I, Rushworth MFS, Passingham RE (2001) Neural correlates of visuomotor associations. Spatial rules compared with arbitrary rules. Exp Brain Res 141:359-369

Touge T, Taylor JL, Rothwell JC (1998) Reduced excitability of the cortico-spinal system during the warning period of a reaction time task. Electroencephalogr Clin Neurophysiol 109:489-495

Van den Hurk P, Mars RB, Van Elswijk G, Hegeman J, Pasman JW, Bloem BR, Toni I (2007) Online maintenance of sensory and motor representations: effects on corticospinal excitability. J Neurophysiol 97:1642-1648

Van Elswijk G, Kleine BU, Overeem S, Stegeman DF (2007) Expectancy induces dynamic modulation of corticospinal excitability. J Cogn Neurosci 19:212-131

Wise SP, Murray EA (2000) Arbitrary associations between antecedents and actions. Trends Neurosci 23:271-273

Young MS, Triggs WJ, Pendergast JF, Keilman KM (2000) The effects of selective response preparation on corticospinal excitability. Cortex 36:19-29 